\title{
Trends in the prevalence of anaemia in Japanese adult women, 1989-2003
}

\author{
Fumi Hayashi ${ }^{1,2, *}$, Nobuo Yoshiike ${ }^{1}$, Katsushi Yoshita ${ }^{3}$ and Kazuo Kawahara ${ }^{2}$ \\ ${ }^{1}$ Centre for Collaborative Research, National Institute of Health and Nutrition, 1-23-1 Toyama Shiniyuku-ku, \\ Tokyo 1628636, Japan: ${ }^{2}$ Department of Health Policy Science, Graduate School of Tokyo Medical and Dental \\ University, Tokyo, Japan: ${ }^{3}$ Nutritional Epidemiology Program, National Institute of Health and Nutrition, Tokyo, \\ Japan
}

Submitted 6 October 2006: Accepted 29 March 2007: First published online 2 July 2007

\begin{abstract}
Objective: There is an increasing concern of anaemia in Japanese women, but no national trend data has existed to date. We analysed long-term national trends of anaemia in adult women.

Design: Secondary analyses of 15 consecutive cross-sectional nationwide surveys conducted during the period 1989-2003. Analyses were based on 5-year intervals (1989-1993, 1994-1998, 1999-2003).

Setting: Japan.

Subjects: The subjects included the National Health and Nutrition Survey of Japan (NHNS-J) population. Analyses were based on 50967 non-pregnant/non-lactating women aged 20 years and over, with complete data sets. We stratified subjects into six age groups (20-29 years, 30-39 years, 40-49 years, 50-59 years, 60-69 years and $70+$ years), and three age groups (20-49 years, 50-69 years, 70+ years) for analyses on residential areas (metropolitan, cities and towns).

Results: Decreases in trends of mean haemoglobin values (intravenous) were significant in all age groups. Changes in the prevalence of anaemia were significant only in women aged 30-39 and 40-49 years. Anaemia (haemoglobin $<12.0 \mathrm{~g} \mathrm{dl}^{-1}$ ) increased from $16.8 \%$ to $20.6 \%$ in women aged $30-39$ years, and from $20.2 \%$ to $26.9 \%$ in women aged $40-49$ years, respectively. Prevalence was highest in women aged 70 years and older in all times, but no significant change was observed.

Conclusions: Haemoglobin values have declined significantly in all ages in the last 15 years. Anaemia was especially significant in women aged 30-39 years and 40-49 years. Our findings suggest that a large number of young women in Japan are at risk of anaemia, so continuous monitoring and controlling efforts of this trend are needed.
\end{abstract}

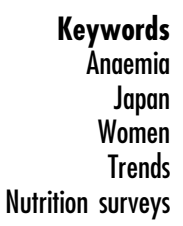

In terms of public health, anaemia or iron deficiency anaemia is the most commonly used indicator of iron deficiency ${ }^{1}$. Iron deficiency anaemia may result from one or more of the following: (1) inadequate dietary iron intake or low iron bioavailability; (2) impaired absorption (e.g. gastric surgery, intestinal malabsorption); (3) increased iron needs during growth or during pregnancy; (4) increased iron loss due to heavy menstrual blood loss or chronic disease $e^{2,3}$. Common causes of anaemia, other than iron deficiency, include other vitamin deficiencies (e.g. vitamin $\mathrm{B}_{12}, \mathrm{~B}_{6}$, folate and $\left.\mathrm{C}\right)^{4}$. Anaemia of chronic disease, a condition accompanied by inflammation, is present in some people, including young children ${ }^{4}$.

The consequences of iron deficiency anaemia or iron deficiency are often deleterious. A review of longitudinal studies indicated that children who were anaemic in early childhood continued to have poor cognition and school performance in middle childhood, compared with nonanaemic children ${ }^{5}$. Severe anaemia during pregnancy is strongly related to increased risks of low birth weight, preterm birth and perinatal mortality ${ }^{6,7}$. Iron deficiency, even without anaemia, is also associated with reduced physical performance and work capacity ${ }^{8}$.

Anaemia is found in approximately two billion people worldwide, with half of all cases caused by a lack of iron? In 2001, iron deficiency anaemia was found to affect $10.3 \%$ of adult women in industrialised countries ${ }^{10}$ and, in the United States in $1998,5 \%$ of women aged $20-49$ years and $2 \%$ of women aged 50 years and older were found to be anaemic ${ }^{2}$. In the United Kingdom, approximately $8 \%$ 
of adult women have iron deficiency anaemia and 11\% have low iron stores without anaemia, according to data from the 2000-01 National Diet and Nutrition Surveys $(\mathrm{NDNS})^{11}$. There is no comparable national data on iron deficiency or iron deficiency anaemia in Japan, other than studies focusing on specific population groups ${ }^{12-14}$. However, the Japanese Red Cross Blood Centre ${ }^{15}$ indicated that the average deferral rate for female donors, due to insufficient blood gravity, increased from $6.4 \%$ to $8.4 \%$ between 1995 and 2005 nationwide. Although low blood gravity is not specific to anaemia, attempts to verify this issue are needed.

The increasing prevalence of underweight women is a major health problem in Japan ${ }^{16}$. Iron deficiency is uncommon among female patients with anorexia nervosa due to increased iron storage secondary to reduced iron losses from amenorrhoea ${ }^{17}$. However, low body mass index (BMI) was an independent risk factor for 'mild iron deficiency' (serum ferritin $<20 \mu \mathrm{gl} \mathrm{l}^{-1}$ and haemoglobin $\geq 120 \mathrm{gl}^{-1}$ ) in premenopausal adult women in New Zealand $^{18}$. Therefore, we suspect that the risk of iron deficiency, and of anaemia, may also be high in Japan. Therefore, we performed long-term trend analyses of anaemia using national data. There are consecutive crosssectional haemoglobin data available for adults, collected between 1989 and 2003. Therefore, we analysed changes in haemoglobin over 15 years, and calculated the prevalence of anaemia using the World Health Organization (WHO) standard ${ }^{10}$. We also evaluated geographical differences in changes in haemoglobin and compared the prevalence of anaemia between three residential area types to determine whether the environment plays a role in anaemia development.

\section{Methods}

\section{Description of the data source}

The National Health and Nutrition Survey of Japan (NHNS-J) is a series of cross-sectional, nationally representative examination surveys conducted by the Ministry of Health, Labour and Welfare ${ }^{19}$. A two-stage sampling technique was used in the NHNS-J. In the first stage, survey districts for the 'Comprehensive Survey of Living Condition of the People on Health and Welfare' were selected using cluster stratified random sampling technique from districts assigned for the nearest population census, which has been conducted every 5 years since 1920. In the second stage for the NHNS-J sampling, a total of 300 survey districts were randomly selected from survey districts for the 'Comprehensive Survey of Living Condition of the People on Health and Welfare', and 5000 households and a total of 15000 individuals in selected households were chosen to participate in NHNS-J each year.

NHNS-J consists of three components: (1) physical examination (anthropometric measurements; blood pressure; blood tests; interview on use of medication, smoking status, alcohol intake and exercise; pedometermeasured physical activity level), (2) dietary intake survey (1-day, semi-weighed food record per household) and (3) dietary habits questionnaire. All physical examinations take place at local centres, and a team of physicians, nurses, dietitians and laboratory technicians are in charge of the assessment. Blood samples are collected from subjects aged 20 years and over, and are drawn at least $3 \mathrm{~h}$ after the last meal. Since 1989, haemoglobin values have been monitored annually, so we performed in-depth analyses on haemoglobin data collected after 1989.

\section{Laboratory procedures and definition of anaemia}

In this study, we evaluated haemoglobin values to determine the prevalence of anaemia. Once intravenous blood is collected, haemoglobin values are determined by an autoanalyser (SE-9000) at a private laboratory commissioned by the government authority. We applied the WHO criteria to define anaemia: haemoglobin concentration of $<12 \mathrm{~g} \mathrm{dl}^{-1}$ for non-pregnant women above 15 years of age ${ }^{10}$. It has been suggested by the National Center for Health Statistics of the Centers for Disease Control and Prevention (CDC) in the United States ${ }^{2}$ that haemoglobin cut-off values should be adjusted for subjects who reside in a high altitude $(\geq 3000 \mathrm{ft}$ ) and for those who smoke cigarettes. However, there is insufficient information regarding the effect of smoking on haemoglobin concentrations in the Japanese and what level of adjustment is appropriate, so we used crude haemoglobin concentrations to evaluate the prevalence of anaemia.

\section{Subjects for the current analyses}

We conducted secondary analyses of 50967 non-pregnant/ non-lactating women aged over 20 years, who participated in the NHNS-J between 1989 and 2003. Subjects with no data on haemoglobin were excluded from the secondary data set. We obtained permission to use the NHNS-J data sets from the relevant government authority prior to the analyses. In order to avoid year-to-year fluctuations and to have adequate sample sizes for subgroup analyses, data were grouped into three time periods (1989-1993, 1994-1998 and 1999-2003). For subgroup analyses, subjects were divided into six age groups (20-29 years; 30-39 years; 40-49 years; 50-59 years; 60-69 years; and 70+ years). In terms of geographical comparisons, we first divided subjects into three age subgroups (20-49 years; 50-69 years; and 70+ years), and then subdivided into three areas ('metropolitan' - 12 major cities and 23 Tokyo Metropolitan Wards with a population of more than 1000000 people; 'cities' - all cities with a population of less than 1000 000; 'towns' - all small towns and villages). This segmentation allows for a better estimation of which group is at higher risk of anaemia. 


\section{Statistics}

Haemoglobin concentrations were expressed as means and standard deviations (SD) for each time period according to age groups and residential areas. The prevalence of anaemia (haemoglobin $<12.0 \mathrm{~g} \mathrm{dl}^{-1}$ ) was expressed as a percentage (\%) for each time period, according to age groups and residential areas.

To evaluate trends in mean haemoglobin levels, we computed regression coefficients for successive survey years using general linear models, coded as 0, 0.2, 0.4, etc., up to 2.8 , for $1989,1990,1991$, etc., up to 2003 . The results are shown as the increment of each parameter per 5 years with 95\% confidence intervals (CIs). To evaluate trends in the prevalence of anaemia across the three time periods, logistic regression analysis was used and the results are shown as the increment in the odds ratio (OR) with 95\% CI (OR for 1989-1993 is 1.00, the reference). For geographical comparisons in the prevalence of anaemia, logistic regression analysis was used to compare values for metropolitan areas and cities with towns within the same age group in each time period. $P<0.05$ was considered to be statistically significant.

The Hosmer-Lemeshow goodness-of-fit was used to assess goodness-of-fit for all models. $P$ for $\chi^{2}$ goodnessof-fit $>0.30$ was considered to be well-fitted for all models. The generalised coefficient of determination statistics, i.e. $R^{2}$, was used to describe the proportion of variability explained by the model. If the value of $R^{2}$ is close to zero, one variable cannot be predictable from other variable. All statistical analyses were performed using SAS software (Version 8.2).

\section{Results}

Table 1 shows changes in mean haemoglobin value in $\mathrm{g} \mathrm{dl}^{-1}$ (with SD) over 15 years, according to the overall and demographic subgroups, i.e. age groups and residential areas. For age groups, the mean haemoglobin values were lowest in subjects aged 40-49 years throughout the three time periods: 12.8 (1.5), 12.6 (1.5) and 12.4 (1.5), respectively. There was a decreasing trend for mean haemoglobin values, with these trends significant in all age groups. In terms of residential areas, statistically significant decreases in mean haemoglobin values were observed in all three areas in all three age subgroups, except for the groups of women aged 70 years and older who resided in metropolitan areas and small towns. The overall change in mean haemoglobin in all subjects aged 20 years and older from 1989 to 2003 was -0.08 (95\% CI $-0.09,-0.07)$, and the decline was statistically significant $(P<0.01)$.

Table 2 shows the prevalence of anaemia in each time period by the overall and demographic subgroups (i.e. age groups and residential areas) and the ORs for changes in the prevalence of anaemia from the logistic regression

Table 1 Trends in mean haemoglobin values in women aged 20 years and over, according to age and residential areas: 1989-2003

\begin{tabular}{|c|c|c|c|c|c|c|c|c|c|}
\hline \multirow[b]{2}{*}{ Variable } & \multicolumn{2}{|c|}{ 1989-1993 } & \multicolumn{2}{|c|}{ 1994-1998 } & \multicolumn{2}{|c|}{ 1999-2003 } & \multirow[b]{2}{*}{ Changes per 5 yearsł } & \multirow[b]{2}{*}{$95 \% \mathrm{Cl}$} & \multirow[b]{2}{*}{$P$-value } \\
\hline & $n$ & Mean (SD) & $n$ & Mean (SD) & $n$ & Mean (SD) & & & \\
\hline Overallt & 21133 & $12.9(1.3)$ & 16558 & $12.9(1.3)$ & 15925 & $12.8(1.2)$ & -0.08 & $-0.09,-0.07$ & $<0.01$ \\
\hline \multicolumn{10}{|c|}{$\begin{array}{l}\text { Demographic subgroup } \\
\text { Age (vears)t }\end{array}$} \\
\hline $20-29$ & 559 & $13.1(1.1)$ & 1743 & $13.0(1.1)$ & 1322 & $12.9(1.1)$ & -0.12 & $-0.16,-0.07$ & $<0.01$ \\
\hline $30-39$ & 4189 & $12.9(1.2)$ & 3025 & $12.8(1.3)$ & 2283 & $12.7(1.2)$ & -0.09 & $-0.12,-0.06$ & $<0.01$ \\
\hline $40-49$ & 5100 & $12.8(1.5)$ & 3863 & $12.6(1.5)$ & 2600 & $12.4(1.5)$ & -0.18 & $-0.21,-0.15$ & $<0.01$ \\
\hline $50-59$ & 4653 & $13.1(1.2)$ & 3948 & $13.2(1.1)$ & 3395 & $13.0(1.1)$ & -0.08 & $-0.11,-0.06$ & $<0.01$ \\
\hline $60-69$ & 3905 & $13.1(1.1)$ & 2233 & $13.1(1.0)$ & 3327 & $13.0(1.0)$ & -0.04 & $-0.06,-0.01$ & $<0.01$ \\
\hline $70+$ & 2727 & $12.7(1.3)$ & 1746 & $12.6(1.2)$ & 2998 & $12.6(1.2)$ & -0.05 & $-0.08,-0.02$ & $<0.01$ \\
\hline \multicolumn{10}{|l|}{ Residential areas } \\
\hline \multicolumn{10}{|l|}{ 20-49 yearst } \\
\hline Metropolitan & 1748 & $12.9(1.3)$ & 1455 & $12.8(1.3)$ & 991 & $12.7(1.2)$ & -0.12 & $-0.17,-0.07$ & $<0.01$ \\
\hline Cities & 5561 & $12.8(1.3)$ & 4873 & $12.8(1.4)$ & 3394 & $12.6(1.3)$ & -0.14 & $-0.16,-0.11$ & $<0.01$ \\
\hline Towns & 2539 & $12.8(1.4)$ & 2303 & $12.8(1.4)$ & 1820 & $12.6(1.4)$ & -0.12 & $-0.16,-0.08$ & $<0.01$ \\
\hline \multicolumn{10}{|l|}{ 50-69 yearst } \\
\hline Metropolitan & 1475 & $13.2(1.1)$ & 1010 & $13.1(1.1)$ & 1012 & $13.0(1.0)$ & -0.07 & $-0.11,-0.03$ & $<0.01$ \\
\hline Cities & 4501 & $13.1(1.1)$ & 3480 & $13.2(1.1)$ & 3724 & $13.1(1.1)$ & -0.07 & $-0.09,-0.04$ & $<0.01$ \\
\hline Towns & 2582 & $13.1(1.1)$ & 1691 & $13.1(1.1)$ & 1986 & $13.1(1.1)$ & -0.05 & $-0.08,-0.02$ & $<0.01$ \\
\hline \multicolumn{10}{|l|}{70 yearst } \\
\hline Metropolitan & 396 & $12.7(1.3)$ & 229 & $12.6(1.1)$ & 390 & $12.6(1.1)$ & -0.06 & $-0.13,0.02$ & 0.14 \\
\hline Cities & 1395 & $12.7(1.3)$ & 867 & $12.6(1.2)$ & 1465 & $12.6(1.2)$ & -0.06 & $-0.10,-0.02$ & 0.01 \\
\hline Towns & 936 & $12.6(1.3)$ & 650 & $12.6(1.2)$ & 1143 & $12.6(1.2)$ & -0.03 & $-0.08,0.02$ & 0.21 \\
\hline
\end{tabular}

SD - standard deviation; $\mathrm{Cl}$ - confidence interval; metropolitan - 12 major cities and 23 Tokyo metropolitan wards with a population more than 1000000 people; cities - all cities with a population less than 1000000 people; towns - all small towns and villages.

Values are shown by means and SD.

† Adjusted for age as a continuous variable.

$\ddagger$ Calculated by general linear model using the raw data from 15 points of observation. Data are expressed as mean haemoglobin changes in value (95\% $\mathrm{Cl}$ ). 
Table 2 Trends in the prevalence of anaemia in women aged 20 years and over, according to age and residential areas: 1989-2003

\begin{tabular}{|c|c|c|c|c|c|c|c|c|c|c|}
\hline \multirow[b]{2}{*}{ Variable } & \multicolumn{3}{|c|}{ Prevalence (\%) } & \multicolumn{3}{|c|}{ Changes per 5 years $\ddagger$} & \multicolumn{3}{|c|}{ Hosmer-Lemeshow's goodness-of-fit§ } & \multirow[b]{2}{*}{$R^{2} \Phi$} \\
\hline & $1989-1993$ & $1994-1998$ & 1999-2003 & OR & $95 \% \mathrm{Cl}$ & $P$ for trend & df & $x^{2}$ & $P$-value & \\
\hline Overallt & 16.5 & 17.6 & 18.6 & 1.08 & $1.05,1.11$ & $<0.01$ & 8 & 68.6 & $<0.01$ & $<0.01$ \\
\hline \multicolumn{11}{|c|}{ Demographic subgroups } \\
\hline \multicolumn{11}{|c|}{ Age (years)† } \\
\hline $20-29$ & 13.4 & 12.8 & 15.4 & 1.12 & $0.98,1.27$ & NS & 8 & 7.7 & 0.47 & 0.01 \\
\hline 30-39 & 16.8 & 18.9 & 20.6 & 1.14 & $1.08,1.21$ & $<0.01$ & 8 & 4.2 & 0.83 & $<0.01$ \\
\hline $40-49$ & 20.2 & 24.5 & 26.9 & 1.20 & $1.14,1.27$ & $<0.01$ & 8 & 26.1 & $<0.01$ & $<0.01$ \\
\hline $50-59$ & 10.9 & 10.6 & 11.7 & 1.06 & $0.99,1.13$ & NS & 8 & 31.6 & $<0.01$ & $<0.01$ \\
\hline $60-69$ & 12.5 & 13.2 & 12.2 & 1.00 & $0.94,1.07$ & NS & 8 & 6.8 & 0.56 & $<0.01$ \\
\hline $70+$ & 25.3 & 26.2 & 26.0 & 1.03 & $0.97,1.09$ & NS & 8 & 11.6 & 0.17 & $<0.01$ \\
\hline \multicolumn{11}{|l|}{ Residential areas } \\
\hline \multicolumn{11}{|l|}{ 20-49 yearst } \\
\hline Metropolitan & $16.8^{\star}$ & 19.4 & $19.1^{*}$ & 1.12 & $1.02,1.23$ & 0.02 & 8 & 7.5 & 0.48 & 0.01 \\
\hline Cities & 18.2 & 20.1 & 22.6 & 1.18 & $1.12,1.24$ & $<0.01$ & 8 & 15.3 & 0.05 & 0.01 \\
\hline Towns & 19.9 & 20.8 & 22.9 & 1.15 & $1.07,1.23$ & $<0.01$ & 8 & 15.7 & 0.05 & 0.01 \\
\hline \multicolumn{11}{|l|}{ 50-69 yearst } \\
\hline Metropolitan & 10.6 & 11.4 & $10.8^{*}$ & 1.04 & $0.92,1.17$ & NS & 8 & 22.0 & 0.01 & $<0.01$ \\
\hline Cities & 11.6 & $11.0^{*}$ & $11.2^{\star \star}$ & 0.99 & $0.93,1.06$ & NS & 8 & 66.8 & $<0.01$ & $<0.01$ \\
\hline Towns & 12.1 & 12.9 & 14.0 & 1.10 & $1.01,1.19$ & 0.02 & 8 & 4.8 & 0.78 & $<0.01$ \\
\hline \multicolumn{11}{|l|}{70 yearst } \\
\hline Metropolitan & 27.0 & 23.6 & 26.9 & 1.00 & $0.86,1.16$ & NS & 8 & 9.0 & 0.34 & 0.02 \\
\hline Cities & 24.0 & 25.3 & 25.2 & 1.04 & $0.96,1.13$ & NS & 8 & 4.3 & 0.83 & 0.03 \\
\hline Towns & 26.6 & 28.3 & 26.6 & 1.02 & $0.93,1.12$ & NS & 8 & 5.9 & 0.66 & 0.03 \\
\hline
\end{tabular}

OR - odds ratio; $\mathrm{Cl}$ - confidence intervals; NS - not significant; metropolitan - 12 major cities and 23 Tokyo metropolitan wards with a population more than 1000000 people; cities - all cities with a population less than 1000000 people; towns - all small towns and villages.

Values are shown in percentage (\%).

t Adjusted for age as a continuous variable.

$\ddagger$ Calculated by logistic models using the raw data from 15 points of observation. Data are expressed as ORs $(95 \% \mathrm{Cl})$. Significance level compared with small towns within the same age group in each time period: ${ }^{\star} P<0.05 ;{ }^{\star \star} P<001$.

$\S$ The Hosmer-Lemeshow goodness-of-fit was used to assess goodness-of-fit for all models.

- The generalised coefficient of determination statictics, i.e. $R^{2}$, was used to describe the proportion of variability explained by the model.

models over 15 years. A higher prevalence of anaemia was observed in women aged $40-49$ years and 70 years and older throughout all time periods, than in other age groups. Among six age groups, the highest OR for changes per 5 years was observed in women aged 40-49 years (OR 1.20, 95\% CI 1.14, 1.27, $P<0.01$ ), followed by women aged 30-39 years (OR 1.14, 95\% CI 1.08, 1.21, $P<0.01$ ). Changes in the prevalence of anaemia in other age groups were not statistically significant. In terms of residential areas, statistically significant changes in metropolitan areas and cities were observed only in women aged 20-49 years, and changes in towns were observed in women aged 20-49 years and 50-69 years. No statistically significant changes in all three areas were observed in women aged 70 years and older. In terms of residential differences on the prevalence of anaemia, the prevalence of anaemia in women aged 20-49 years living in metropolitan areas was statistically lower than in towns in 1989-1993 and 1999-2003. Statistically significant lower prevalence of anaemia was observed in women aged 50-69 years living in cities and cities and metropolitan areas than in towns in 1994-1998 and 1999-2003, respectively. No residential differences were observed in women aged 70 years and older throughout all three time periods. The Hosmer and Lemeshow goodness-of-fit tests indicated the models categorised by demographic sub- groups fitted relatively well ( $P$-values $>0.03$ ), except for some subgroups. The $R^{2}$ values for all models indicated that the explanatory variables in the models have little contribution.

\section{Discussion}

WHO proposes that the public health significance of anaemia be classified on the prevalence estimated from blood haemoglobin levels or haematocrit ${ }^{10}$, with proposed levels of significance as follows: 'normal' ( $\leq 5 \%)$, 'mild' (5.0 to 19.9\%), 'moderate' (20.0-39.9\%), and 'severe' ( $\geq 40 \%)$. Our results clearly demonstrate a level of significance in Japanese women is either 'mild' or 'moderate', according to the WHO classification ${ }^{10}$. For the most recent time period (1999-2003), anaemia was particularly prominent in women aged 40-49 years (26.9\%), in women aged 70 years and older (26.0\%) and in women aged 30-39 years (20.6\%). A lower prevalence of anaemia was found among women aged 50-59 years and 60-69 years, but the level remained high enough to warrant attention. Most importantly, the prevalence has been increasing.

In this study, we also observed higher prevalence of anaemia in towns, compared to metropolitan and cities, 
in all three periods, except for elderly women aged 70 years and older. Back in the 1960s and until the 1980s, anaemia was the major public health problem among Japanese women, especially among those in villages. Several factors were considered as possible reasons for higher prevalence of anaemia in villages compared to cities or towns. For example, undernutrition, higher number of pregnancies, overworking and parasite infections were discussed as possible risk factors ${ }^{20}$. Today, however, there is not much difference in nutrition and health environments between cities and villages due to the nationwide economic growth. Overnutrition, represented by increased prevalence of obesity, is now greater in towns than in metropolitan areas ${ }^{21}$. Further studies are needed to examine why the prevalence of anaemia remains high in towns as we observed in the present study, and whether other developed and Asian countries have similar situations to ours.

There are many causes of anaemia in a population. However, in terms of public health, iron deficiency is the most important cause of nutritional anaemia worldwide. Low iron intake may be a significant cause in Japanese women, especially in young women. The Estimated Average Requirement (EAR) for iron is $9.0 \mathrm{mg}$ for menstruating adult women and $5.5 \mathrm{mg}$ for non-menstruating adult women ${ }^{22}$. The actual average intake (and its $\mathrm{SD}$ in $\mathrm{mg}$ ) in women aged 20-29 years, 30-39 years, 40-49 years, 50-59 years, 60-69 years and over 70 years, according to NHNS-J 2003, was 7.0 (2.9), 7.3 (6.0), 8.9 (20.1), 8.7 (3.6), 9.3 (3.9) and 8.1 (3.5), respectively ${ }^{23}$. These data show that the prevalence of low iron intake might be high in young Japanese women. Second, diseases related to increased iron losses, such as 'menorrhagia' or leiomyoma, may be common in Japanese women. A 4-year follow-up study of 9506125 - to 44-yearold premenopausal women in the United States, estimated the incidence of uterine leiomyoma at 12.8 per 1000 woman-years ${ }^{24}$. Although estimates of the incidence among Japanese premenopausal women vary between studies, approximately $30 \%$ of all women of reproductive age are susceptible to uterine leiomyoma in Japan ${ }^{25}$. The aetiology of anaemia (e.g. insufficient dietary iron intakes, high blood iron loss) needs further evaluation with the use of other indicators of iron status.

A major strength of this study is that this is the first study identifying prevalence and risks of iron deficiency and iron deficiency anaemia in young Japanese women using a large nationwide sample. However, there are some limitations that need to be highlighted. First, data are not obtained using a simple random sample. Rather, a stratified cluster sampling design is used to select participants. The smallest random sampling unit is a district, not an individual, and the number of households and size of households differ from district to district. Sampling design is consistent each year so we assume that there is no sampling effect on the equality of subgroups in this study. However, the response rate for the NHNS-J has declined recently; therefore, the estimates may differ greatly when they are calculated with the correct weight than when they are calculated without being weighted at all. In this study, we used non-weighted data for all analyses. Further assessment is needed once sample weights and imputation for non-response are fully taken into account in the methodology of NHNS-J in future.

A definition of anaemia in this study is another consideration. We estimated anaemia with a single measure, which was haemoglobin concentration below the WHOestablished cut-off level ${ }^{10}$. The haemoglobin level drops when anaemia is present; however, such a drop cannot be used to distinguish between nutritional anaemia (such as iron deficiency) and anaemia from other causes. Binkin and Yip $^{26}$ reported that using haemoglobin to screen for iron deficiency detected only $37 \%$ of iron-deficient women in the United States. In order to detect iron deficiency as a risk for nutritional anaemia, WHO/CDC experts recommend using haemoglobin and ferritin (and the transferrin receptor where infection is common) ${ }^{10}$. The NHNS-J started monitoring ferritin in 2004. Therefore, we will review the prevalence of, and secular trends in, iron deficiency in the population once we have an adequate sample size. Another limitation is that we do not know the probability of a woman being at risk of chronic disease or menstruating. Lastly, the Hosmer-Lemeshow goodness-of-fit tests did not fit well in all of the models we tested and the $R^{2}$ values indicated that the explanatory variables used in all models had little contribution. Therefore, future research should focus on studying additional constructs that might explain changes in prevalence of anaemia in the Japanese women. None the less, the results must be interpreted with caution and further research is needed; still, this study provides baseline data for future public policy making and nutrition monitoring for high-risk subgroups (e.g. middle-aged women, women residing in towns).

In summary, this is the first study reporting long-term trends of anaemia in adult Japanese women, using data sets collected nationwide. Although some considerations are needed in interpretation of the findings, the study indicated that middle-aged women, especially those aged 40-49 years, are at a higher risk for developing anaemia. Data also indicated that there may be additional risks for women living in towns. Comprehensive efforts to reduce risks of anaemia are needed, and nutrition monitoring systems useful to collect the nationally representative samples also need to be developed.

\section{Acknowledgments}

Sources of funding: This study was funded by Research on Health Science (the Ministry of Health, Labour and Welfare). 
Conflict of interest declaration: None of the authors had a personal or financial conflict of interest.

Authorship responsibilities: F.H. was responsible for the conception of the study, with assistance from N.Y. and K.K.; N.Y. obtained funding; F.H. analysed and interpreted the data, with assistance from N.Y. and K.K.; K.Y. provided statistical expertise; F.H. wrote the manuscript; F.H. was responsible for review and revision of the manuscript, with assistance from N.Y., K.Y. and K.K.

Acknowledgments: The authors thank Dr Yokoyama (National Institute of Public Health) for statistical advice.

\section{References}

1 Morland K, Wing S, Roux AD. The contextual effect of the local food environment on residents' diets: the atherosclerosis risk in communities study. American Journal of Public Health 2002; 92: 1761-7.

2 Centers for Disease Control and Prevention. Recommendations to prevent and control iron deficiency in the United States. Morbidity and Mortality Weekly Report 1998; 47: 6-10.

3 Goddard AF, McIntyre AS, Scott BB, for the British Society of Gastroenterology. Guidelines for the management of iron deficiency anaemia. Gut 2000; 46: iv1-5.

4 The Iron Disorders Institute. Guide to Anaemia. Tennessee: Cumberland House Publishing Inc., 2003.

5 McGregor S, Ani C. A review of studies on the effect of iron deficiency on cognitive development in children. Journal of Nutrition 2001; 131(2S-2): 649S-68S.

6 Lee HS, Kim MS, Kim MH, Kim YJ, Kim WY. Iron status and its association with pregnancy outcome in Korean pregnant women. European Journal of Clinical Nutrition 2006; 60: $1130-5$.

7 Rasmussen KM. Is there a causal relationship between iron deficiency or iron-deficiency anaemia and weight at birth, length of gestation and perinatal mortality? Journal of Nutrition 2001; 131(2S-2): 590S-601S.

8 Hass JD, Brownlie T. Iron deficiency and reduced work capacity: a critical review of the research to determine a causal relationship. Journal of Nutrition 2001; 131(2S-2): 676S-90S.

9 de Benoist B. WHO/CDC Expert Consultation Agrees on Best Indicators to Assess Iron Deficiency, a Major Cause of Anaemia [online], May 2004. Available at http:// www.who.int/mediacentre/news/notes/2004/anaemia/en/. Accessed 28 August 2006.

10 World Health Organization (WHO). Iron Deficiency Anaemia: Assessment, Prevention, and Control - A Guide for Programme Managers. Geneva: WHO, 2001.

11 Fairweather-Tait SJ. Iron nutrition in the UK: getting the balance right. Proceedings of the Nutrition Society 2004; 63: 519-28.
12 Maeda M, Yamamoto M, Yamauchi K. Prevalence of anaemia in Japanese adolescents: 30 years' experience in screening for anaemia. International Journal of Hematology 1999; 69: 75-80.

13 Ookura M, Kawabata T, Koide M, Kamiyama K, Amamiya K, Miyamura R, et al. Prevalence of iron deficiency of anaemia in females residing Saitama city: results of 2002 Health Check. Saitamaken Igakkai Zasshi 2004; 38: 611-16 [in Japanese].

14 Uchida T, Kawachi Y, Sakamoto Y, Igaki T, Ogasawara N, Kariyone S, et al. Prevalence and pathogenesis of iron deficiency in Japanese women (1981-1991). Rinsho Ketsueki 1992; 33: 1661-5 [in Japanese].

15 Japan Red Cross Society Blood Division. Annual Report of the Blood Project in 2005 [online], 2005. Available at http:// www.jrc.or.jp/active/blood/pdf/2005toukei.pdf. Accessed 29 September 2006 [in Japanese].

16 Takimoto H, Yoshiike N, Kaneda F, Yoshita K. Thinness among young Japanese women. American Journal of Public Health 2004; 94: 1592-5.

17 Kennedy A, Kohn M, Lammi A, Clarke S. Iron status and haematological changes in adolescent female inpatients with anorexia nervosa. Journal of Paediatrics and Child Health 2004; 40: 430-2.

18 Heath AL, Skeaff CM, Williams S, Gibson RS. The role of blood loss and diet in the aetiology of mild iron deficiency in premenopausal adult New Zealand women. Public Health Nutrition 2001; 4: 197-206.

19 Ministry of Health, Labour and Welfare. Annual Report of the National Health and Nutrition Survey in 2002. Tokyo: Ministry of Health, Labour and Welfare, 2004 [in Japanese].

20 Owada K, Tanaka H, Hattori A, Ueda Y, Date C, Tsue H, et al. Multivariate data analysis of causative factors of anaemia of women living in the rural district. Nipon Koshu Eisei Zasshi 1974; 21: 379-86 [in Japanese].

21 Yoshiike N, Seino F, Tajima S, Arai Y, Kawano M, Furuhata $\mathrm{T}$, et al. Twenty-year changes in the prevalence of overweight in Japanese adults: The National Nutrition Survey 1976-95. Obesity Reviews 2002; 3: 183-90.

22 Ministry of Health, Labour and Welfare. Dietary Reference Intakes for Japanese, 2005. Tokyo: Ministry of Health, Labour and Welfare, 2005 [in Japanese].

23 Ministry of Health, Labour and Welfare. Annual Report of the National Health and Nutrition Survey in 2003. Tokyo: Ministry of Health, Labour and Welfare, 2005 [in Japanese].

24 Marshall LM, Spiegelman D, Barbieri RL, Goldman MB, Manson JE, Colditz GA, et al. Variation in the incidence of uterine leiomyoma among premenopausal women by age and race. Obstetrics and Gynecology 1997; 90: 967-73.

25 Takamatsu S, Nakagawa H, Kitaoka Y, Kishi K, Tominaga E, Tanigaki $\mathrm{N}$, et al. Uterine myoma and endometriosis. Current Therapy 2006; 24: 73-7 [in Japanese].

26 Binkin NJ, Yip R. When is anaemia screening of value in detecting iron deficiency? In: Hercberg S, Galan P, Dupin H, eds. Recent Knowledge on Iron and Folate Deficiencies in the World. Colloque INSERM, Vol. 197. Paris: INSERM, 1990; $137-46$. 